\title{
A Tunable Plasmonic Wide Flat-Top Band-Pass Filter based on Trapezoid Resonator at Near-Infrared
}

\author{
Seyed Morteza Ebadi (1) *, Student Member, OSA, and Jonas Örtegren (1) \\ ${ }^{(1)}$ Department of Natural Sciences, Mid Sweden University, SE-85170 Sundsvall, Sweden \\ seyed-morteza.ebadi@miun.se
}

\begin{abstract}
A miniaturized and efficient tunable wide flat-top band-pass filter at NIR is proposed in a MIM plasmonic wa veguide. Furthermore, numerical results illustrate that tunability is rea dily attained by varying length of right-angle trapezoid resonator.
\end{abstract}

\section{Introduction}

Surface plasmon polaritons (SPPs), provide a platform that can incorporate electronics and photonics circuits on the same chip, leading to significant reduction in size, energy consumption, and higher efficiency. The plasmonic-based devices, carrying both optical and electrical signals and offering to manipulate and confine light at the nanometer scale, have attracted considerable a mount of attention. Indeed, there are two waveguide configurations based on the SPPs; metal-insulator-metal (MIM) and insulator-metal-insulator (IMI), where the first type is favored for photonic integrated circuits (PICs), due to its great mode confinement, while its losses are regarded to be within the accepted level [1-3]. Therefore, various optical structures based on the MIM plasmonic waveguides including highly-sensitive biosensors for detecting human blood group [4], a compact all-optical plasmonic switch [5], light absorption enhancement [6], and so forth have been suggested and numerica lly investigated. Among them, optical filters that are an integral part in many components and applications ranging from wavelength division multiplexing (WDM), machine vision systems, to laser experiments, and light detection and ranging (LIDAR), play a vitally important role in the performance of the entire system. Hence, we design and propose a new compact and efficient tunable wide flattop band-pass filter at the near-infrared (NIR).

\section{Device Structure and Numerical Results}
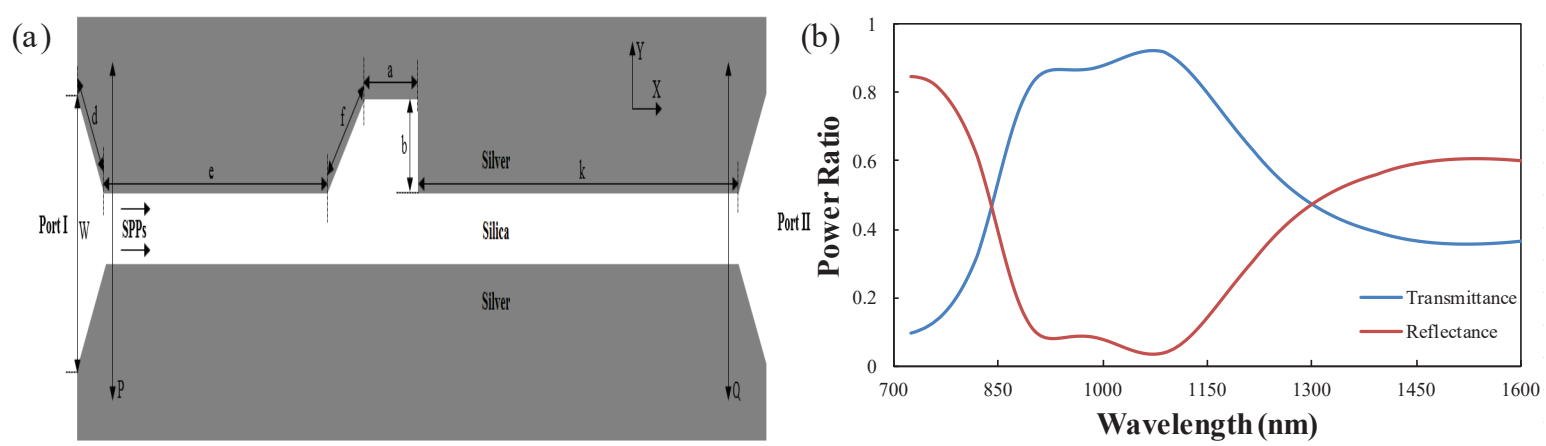

Fig. 1. (a) Schematic configuration of the suggested wide flat-top band-pass filter at NIR. (b) Transmittance and reflectance spectra of the wavelength filter for $\mathrm{W}=80 \mathrm{~nm}, \mathrm{~d}=40 \mathrm{~nm}, \mathrm{e}=160 \mathrm{~nm}, \mathrm{f}=\mathrm{b}=35 \mathrm{~nm}, \mathrm{a}=50 \mathrm{~nm}$, and $\mathrm{k}=185 \mathrm{~nm}$.

Figure 1. (a) schematically illustrates the device configuration of the proposed tunable plasmonic wide flat-top bandpass filter at NIR. As shown in Fig. 1(a), the device structure consists of an insulator layer that is chosen to be Silica, whose dielectric constant is 2.5 , which is sandwiched between two metallic layers, assumed to be silver. Experimental data provided by Johnson and Christy [7] was used in the simulation. The wide band-pass is realized by an efficient coupling between the bust waveguide and the right-angle trapezoid resonator. In the following, we describe the steps that were taken to obtain the simulations results; the fundamental TM mode of the MIM waveguide is excited by a dipole source. The light is launched from the left port, Port I, as displayed in Fig. 1. (a). The gird sizes are set to be 7 $\mathrm{nm} \times 7 \mathrm{~nm}$ along $\mathrm{x}$ and $\mathrm{y}$, which ensures accuracy in the results. Next, two power monitors $\mathrm{P}$ and $\mathrm{Q}$, are set to record the incident and transmitted power in the device, and the transmittance can therefore be specified by as T=Pout/Pin. A full-wave EM tool, CST MWS, with frequency domain solver which is based on the finite element method (FEM) is utilized to study the optical properties of the filter [8]. The simulation result of the transmittance and reflectanceprofiles of the filter is exhibited in Fig. 1. (b) for the physical parameters $\mathrm{W}=80 \mathrm{~nm}$, as the width of the input port, $\mathrm{d}=40 \mathrm{~nm}$, illustrating the distance from the input port to bus waveguide, $\mathrm{e}=160 \mathrm{~nm}$, as the distance from the left bus waveguide 
to the right-angle trapezoid resonator, $\mathrm{f}=\mathrm{b}=35 \mathrm{~nm}$, accounts for the lengths opposite to the right side and the right-angle of the trapezoid, $\mathrm{a}=50 \mathrm{~nm}$, displays the width of the resonator, and $\mathrm{k}=185 \mathrm{~nm}$, indica tes the distance from the trapezoid to the right bus waveguide. Consequently, one can see that a wide flat-top band-pass occurs from wavelengths 892.4 $\mathrm{nm}$ to $1147.5 \mathrm{~nm}$, with an efficiency that is higher than $80 \%$, while the absorption remains less than $6.5 \%$ within that range.

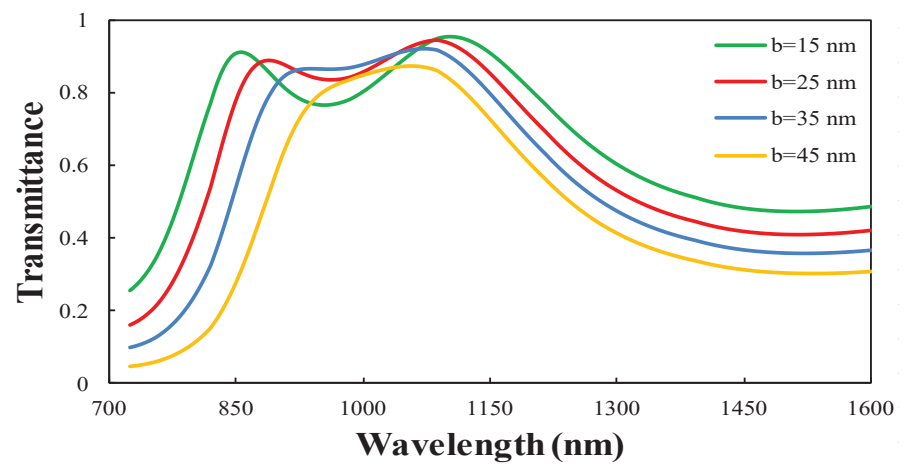

Fig. 2. The transmission spectra as a function of the wavelength for theplasmonic wide flat-top band-pass filter with different lengths of the right-angle trapezoid resonator.

Figure 2 demonstrates the numerical results of the suggested flat-top band-pass filter as a function of wavelength for different lengths of the right-angle trapezoid resonator, while other structural parameters are kept the same as in Fig. 1. (a). The transmission profile illustrates that by increasing the length of the resonator, not only does the flat-top band-pass move to longer wavelengths, but it a lso flattens the pass-band. Indeed, with $b=25 \mathrm{~nm}$, the flat-top bandwidth with an efficiency of at least $80 \%$ goes from wavelengths $855 \mathrm{~nm}$ to $1170 \mathrm{~nm}$, and with $\mathrm{b}=45 \mathrm{~nm}$ the wide band-pass is reduced to the wavelength interval $954.5 \mathrm{~nm}-1119.4 \mathrm{~nm}$. We therefore have shown that by simply adjusting a structural parameter of the filter, the flat-top band-pass spectrum can be readily tuned, which offers more degrees of freedom to design and fabricate na nostructures.

\section{Conclusion}

In conclusion, a highly-compact and efficient tunable wide flat-top band-pass based on MIM pla smonic waveguide at NIR is suggested, and simulation results demonstrate that the transmission spectrum can be modulated by tuning a structural parameter. As a result, due to its miniaturized size, good out-of-band rejection, and high-efficiency compared to similar studies [9-11], it may find applications in tunable optical wireless communication systems and in on-chip integration.

\section{References}

[1] Halas, Naomi J., et al. "Plasmons in strongly coupled metallic nanostructures." Chemical reviews 111.6 (2011): 3913-3961.

[2] Zia, Rashid, et al. "Plasmonics: the next chip-scale technology." Materials today", 9.7-8 (2006): 20-27.

[3] Stockman, Mark I., et al. "Roadmap on plasmonics." Journal of Optics 20.4 (2018): 043001.

[4] Amoosoltani, Narjes, et al. "A plasmonic nano-biosensor based on two cons ecutive disk resonators and unidirectional reflectionless propagation effect." IEEE Sensors Journal 20.16 (2020): 9097-9104.

[5] Negahdari, Roozbeh, Esmat Rafiee, and Farzin Emami. "Realization of all-optical plasmonic MIM split square ring resonator switch." Optical and Quantum Electronics 51.7 (2019): 1-14.

[6] Luo, Xin, et al. "Enhanced dual-band absorption of molybdenum disulfide using a plasmonic perfect absorber." Optics express 26.9 (2018): $11658-11666$.

[7] P. B. Johns on and R.-W. Christy, "Optical constants of the noble metals," Physical review B, vol. 6, no. 12, p. 4370, 1972.

[8] S. M. Ebadi, et al, "A Multipurpose and Highly-Compact Plasmonic Filter Based on Metal-Insulator-Metal Waveguides," IEEE Photonics Journal, vol. 12, no. 3, pp. 1-9, June 2020.

[9] Deng, Haidong, Yuqi Yan, and Yi Xu. "Tunable flat-top bandpass filter based on coupled resonators on a graphene sheet." IEEE Photonics Technology Letters 27.11(2015): 1161-1164.

[10] Yang, Liu, et al. "Compact wideband plasmonic filter with flat-top transmission response based on corrugated metal-insulator-metal ring resonator." Scientific reports 7.1 (2017): 1-10.

[11] Ebadi, Seyed Morteza, and Jonas Örtegren. "A Reconfigurable and Ultra-Compact Plasmonic Filter based on MIM Waveguides at Optical Channels." NovelOptical Materials and Applications. Optical Society of America, 2020. 